Acknowledgements. The author is pleased to acknowledge review of the manuscript of this paper and helpful suggestions thereto by Dr. H. M. Trent of the U. S. Naval Research Laboratory and Dr. D. E. Spencer of the University of Connecticut.

\title{
REFERENCES
}

1. E. T. Whittaker and G. N. Watson, A Course of Modern Analysis, (MacMillan Co., New York, 1948), American edition

2. H. Bateman, Partial Differential Equations of Mathematical Physics, (Dover Publications, Inc., New York, 1944), first American edition

3. E. Kamke, Differentialgleichungen Lösungsmethoden und Lösungen, (Chelsea Publishing Co., New York, 1942)

4. A. G. Webster, Partial Differential Equations of Mathematical Physics, (Dover Publications, Inc., 1955), reprint of second corrected edition

5. A. Sommerfeld, Partial Differential Equations in Physics, (Academic Press, New York, 1949)

6. M. G. Salvadori and R. J. Schwarz, Differential Equations in Engineering Problems, (Prentice-Hall, Englewood Cliffs, New Jersey, 1954)

7. P. Moon and D. E. Spencer, Foundations of Electrodynamics, (D. Van Nostrand Co., Inc., Princeton, New Jersey, 1960)

8. P. Moon and D. E. Spencer, Field Theory for Engineers, (D. Van Nostrand Co., Inc., Princeton, New Jersey, 1961)

9. P. Moon and D. E. Spencer, J. Franklin Inst., 256 (1953) 551

\section{ON LINEAR EQUATIONS OF ANISOTROPIC ELASTIC PLATES*}

\section{BY YI-YUAN YU (Polytechnic Institute of Brooklyn)}

1. Introduction. In a previous paper [1] a generalized Hamilton principle and the associated variational equation of motion are presented for finite elastic deformations. The principle is a generalization of the ordinary Hamilton principle in which only variations of the displacements are admitted. It is also a generalization of the variational principle in elastostatics due to $\mathrm{Hu}[2]^{* *}$ and Washizu [3], and is similar to the latter in that in both principles variations of the displacements, strains, and stresses are taken independently and simultaneously. Various complete systems of equations of nonlinear elastodynamics have been shown to be obtainable as the Euler equations of the generalized Hamilton principle.

In this note the generalized Hamilton principle and the associated variational equation of motion for linear and anisotropic elastic plates are deduced from their counterpart in general elasticity theory given in the previous paper [1], through expansion of the displacement and strain in infinite power series in the manner of Cauchy and Mindlin $[4,5]$ and by carrying out the integration in the thickness direction of the plate. The Euler equations of the variational principle then yield the complete system of plate equations of all orders as were obtained by Mindlin by a different procedure. Some zeroorder and first-order equations for an isotropic plate have been derived before [1] from the generalized variational equation of motion.

${ }^{*}$ Received February 3, 1964. This research was supported by the Air Force Office of Scientific Research of the Office of Aerospace Research, under Contract No. AF 49(638)-1290.

**The author is indebted to Professor C. S. Hsu for drawing attention to Hu's work. 
2. Generalized Hamilton principle. The generalized Hamilton principle [1] takes the following form in the linear theory of elasticity:

$$
\delta \int_{t_{0}}^{t_{2}} L d t=\delta \int_{t_{0}}^{t_{1}}(T-U+W) d t=0,
$$

where

$$
\begin{aligned}
T & =\int \frac{1}{2} \rho u_{i} u_{i} d V \\
U & =\int\left[\frac{1}{2} \sigma_{i j}\left(u_{i, i}+u_{i_{i}}\right)-\sigma_{i j} \epsilon_{i j}+\frac{1}{2} c_{i j k l} \epsilon_{i j} \epsilon_{k l}\right] d V \\
W & =\int f_{i} u_{i} d V+\int p_{i}^{*} u_{i} d S_{p}+\int p_{i}\left(u_{i}-u_{i}^{*}\right) d S_{u}
\end{aligned}
$$

In (2) $\rho$ is the density, $u_{i}$ the displacement vector, $\epsilon_{i j}$ the strain tensor, $\sigma_{i i}$ the stress tensor, $c_{i j k l}$ the elastic stiffness tensor, $f_{i}$ the body force, and $p_{i}$ the surface traction. $V$ indicates the volume of the body, $S_{p}$ the part of the surface $S$ of the body on which the traction is prescribed, and $S_{u}$ the part of $S$ on which the displacement is prescribed. Finally, $t_{0}$ and $t_{1}$ are two instants of time, and the dot indicates differentiation with respect to the time $t$, and the asterisk denotes a prescribed quantity.

When variations of the displacements, strains, and stresses are taken independently and simultaneously, (1) yields

$$
\begin{aligned}
& \int_{t_{0}}^{t_{2}} d t \int\left(\sigma_{i i, i}+f_{j}-\rho \ddot{u}_{i}\right) \delta u_{j} d V-\int_{t_{0}}^{t_{2}} d t \int\left(\sigma_{i j} \nu_{i}-p_{i}^{*}\right) \delta u_{i} d S_{p} \\
& \quad+\int_{t_{0}}^{t_{1}} d t \int\left(\sigma_{i i}-c_{i j k l} \epsilon_{k l}\right) \delta \epsilon_{i j} d V+\int_{t_{0}}^{t_{2}} d t \int\left[\epsilon_{i i}-\frac{1}{2}\left(u_{i, i}+u_{i, i}\right)\right] \delta \sigma_{i i} d V \\
& \quad+\int_{t_{0}}^{t_{2}} d t \int\left(u_{i}-u_{i}^{*}\right) \delta p_{i} d S_{u}=0,
\end{aligned}
$$

which is the generalized variational equation of motion associated with, and completely equivalent to, the variational principle in (1). The Euler equations written from (3) give, respectively, the stress equations of motion, traction boundary conditions, stress-strain relations, strain-displacement relations, and displacement boundary conditions, which constitute the complete system of equations of linear elasticity.

3. Plate equations. The plate is referred to rectangular coordinates $x, y, z$, with $x$ and $y$ in the middle plane and the faces at $z= \pm h$. As in Mindlin's work $[4,5]$, we write

$$
u_{i}=\sum_{n=0}^{\infty} z^{n} u_{i}^{(n)}, \quad \epsilon_{i i}=\sum_{n=0}^{\infty} z^{n} \epsilon_{i i}^{(n)}, \quad \sigma_{i i}^{(n)}=\int_{-h}^{h} \sigma_{i i} z^{n} d z,
$$

where $u_{i}^{(n)}, \epsilon_{i j}^{(n)}, \sigma_{i j}^{(n)}$ are independent of the thickness coordinate $z$ and are the $n$ th-order components of the plate-displacement, -strain, and -stress, respectively.

We substitute the series expansions of $u_{i}$ and $\epsilon_{i i}$ from (4) into (2), carry out the integration with respect to $z$, and introduce $\sigma_{i j}^{(n)}$ according to (4). The results are

$$
T=\int \frac{1}{2} \rho \sum_{m=0}^{\infty} \sum_{n=0}^{\infty} H_{m n} u_{i}^{\cdot(m)} u_{i}^{\cdot(n)} d A
$$




$$
\begin{aligned}
U=\int\left[\frac{1}{2} \sum_{n=0}^{\infty} \sigma_{i j}^{(n)}\left\{u_{i, i}^{(n)}+u_{i, i}^{(n)}+(n+1)\left(\delta_{z i} u_{i}^{(n+1)}+\delta_{i z} u_{i}^{(n+1)}\right)\right\}\right. \\
\left.-\sum_{n=0}^{\infty} \sigma_{i j}^{(n)} \epsilon_{i j}^{(n)}+\frac{1}{2} c_{i j k l} \sum_{m=0}^{\infty} \sum_{n=0}^{\infty} H_{m n} \epsilon_{i j}^{(n)} \epsilon_{k l}^{(m)}\right] d A \\
W=\int \sum_{n=0}^{\infty} f_{i}^{(n)} u_{i}^{(n)} d A+\int \sum_{n=0}^{\infty} P_{i}^{*(n)} u_{i}^{(n)} d A_{p}+\int \sum_{n=0}^{\infty} p_{i}^{*(n)} u_{i}^{(n)} d C_{p} \\
+\int \sum_{n=0}^{\infty} P_{i}^{(n)}\left(u_{i}^{(n)}-u_{i}^{*(n)}\right) d A_{u}+\int \sum_{n=0}^{\infty} p_{i}^{(n)}\left(u_{i}^{(n)}-u_{i}^{*(n)}\right) d C_{u},
\end{aligned}
$$

where

$$
\begin{aligned}
& H_{m n}=\left\{\begin{array}{ccc}
2 h^{m+n+1} /(m+n+1) & \text { for } & \text { even } m+n, \\
0 & \text { for } & \text { odd } m+n,
\end{array}\right. \\
& f_{i}^{(n)}=\int_{-h}^{h} f_{i} z^{n} d z, \\
& p_{i}^{(n)}=\int_{-h}^{h} p_{i} z^{n} d z, \\
& P_{i}^{(n)}=\left[p_{i} z^{n}\right]_{z=h}+\left[p_{i} z^{n}\right]_{z=-h} .
\end{aligned}
$$

In addition, $\delta_{z i}$ and $\delta_{i s}$ in (5) are Kronecker deltas, $A_{p}$ and $A_{u}$ those parts of the plan area $A$ of the plate, and $C_{p}$ and $C_{u}$ those parts of the edge of the plate, on which traction and displacement, respectively, are prescribed. The generalized Hamilton principle (1) becomes that for the plate when (5) is used instead of (2).

The generalized variational equation of motion for the plate may now be derived from (1) and (5) or, more directly, by carrying out the integration with respect to $z$ in (3) together with the use of (4). The result is

$$
\begin{aligned}
& \int_{t_{0}}^{t_{1}} d t \int \sum_{n=0}^{\infty}\left(\sigma_{i j, i}^{(n)}-n \sigma_{z i}^{(n-1)}+P_{i}^{*(n)}+f_{i}^{(n)}-\rho \sum_{m=0}^{\infty} H_{m n} u_{i}^{(m)}\right) \delta u_{i}^{(n)} d A \\
& +\int_{t_{0}}^{t_{1}} d t \int \sum_{n=0}^{\infty}\left(\sigma_{i j}^{(n)}-c_{i j k l} \sum_{m=0}^{\infty} H_{m n} \epsilon_{k l}^{(m)}\right) \delta \epsilon_{i j}^{(n)} d A \\
& +\int_{t_{0}}^{t_{1}} d t \int \sum_{n=0}^{\infty}\left\{\epsilon_{i j}^{(n)}-\frac{1}{2}\left[u_{i, j}^{(n)}+u_{i, i}^{(n)}+(n+1)\left(\delta_{z i} u_{i}^{(n+1)}+\delta_{i z} u_{j}^{(n+1)}\right)\right]\right\} \delta \sigma_{i j}^{(n)} d A \\
& +\int_{t_{0}}^{t_{1}} e^{7} t \int \sum_{n=0}^{\infty}\left(u_{i}^{(n)}-u_{i}^{*(n)}\right) \delta P_{i}^{(n)} d A_{u} \\
& -\int_{t_{0}}^{t_{1}} d t \int \sum_{n=0}^{\infty}\left[\left(\sigma_{n n}^{(n)}-p_{n}^{*(n)}\right) \delta u_{n}^{(n)}+\left(\sigma_{n s}^{(n)}-p_{s}^{*(n)}\right) \delta u_{s}^{(n)}+\left(\sigma_{n z}^{(n)}-p_{z}^{*(n)}\right) \delta u_{z}^{(n)}\right] d C_{p} \\
& +\int_{t_{0}}^{t_{2}} d t \int \sum_{n=0}^{\infty}\left[\left(u_{n}^{(n)}-u_{n}^{*(n)}\right) \delta p_{n}^{(n)}+\left(u_{s}^{(n)}-u_{s}^{*(n)}\right) \delta p_{s}^{(n)}+\left(u_{z}^{(n)}-u_{z}^{*(n)}\right) \delta p_{z}^{(n)}\right] d C_{u}=0
\end{aligned}
$$

where the subscripts $n$ and $s$ denote directions normal and tangential, respectively, to the plate contour. 
The Euler equations for (6) constitute the complete system of equations of an anisotropic plate. It is noted that the term $P_{i}^{*(n)}$ in the stress equation of motion given by (6) is a function of the surface traction. This automatically takes care of the traction boundary conditions in the interior of the plate, while the integral over $A_{u}$ in (6) yields the displacement boundary conditions in the interior of the plate. In fact, (6) yields in a simple manner all conditions at the edge as well as in the interior of the plate for equations of all orders.

It is noted that the plate equations written from (6) are essentially those given by Mindlin $[4,5]$, who used the ordinary variational equation of motion in deriving the stress equations of motion and Neumann's method in deriving the boundary conditions. Mindlin's procedure is thus also justified from the variational point of view.

There is no difficulty in extending the present treatment of plates to shells. The results are reported elsewhere [6].

\section{REFERENCES}

1. Yi-Yuan Yu, Generalized Hamilton's principle and variational equation of motion in nonlinear elasticity theory, with application to plate theory, J. Acoustical Soc. Amer. 36 (1964) 111

2. Hai-Chang $\mathrm{Hu}, \mathrm{On}$ some variational principles in the theory of elasticity and the theory of plasticity, Acta Physica Sinica 10 (1954) 259, also Scientia Sinica 4 (1955) 33

3. K. Washizu, On the variational principles of elasticity and plasticity, M. I. T. Aeroelastic Structures Res. Lab. Tech. Report 25.18 (1955)

4. R. D. Mindlin, An introduction to the mathematical theory of vibrations of elastic plates, U. S. Army Signal Corps, Fort Monmouth, N. J., 1955

5. R. D. Mindlin, High frequency vibrations of crystal plates, Q. Appl. Math. 19 (1961) 51

6. Yi-Yuan Yu, On linear equations of isotropic elastic plates and shells, Scientific Report No. 2, Polytechnic Institute of Brooklyn, Dept. of Mech. Engg., 1964

\section{A CORRESPONDENCE PRINCIPLE FOR VISCOELASTIC WAVE PROPAGATION*}

By I. HERRERA, (University of Mexico) AND M. E. GURTIN, (Brown University)

Introduction. It is a well known result of classical linear elasticity theory that the speed of propagation $U$ of an acceleration wave, at a point $x$ in an elastic solid of elasticity tensor $c_{i j k l}(x)$ and density $\rho(x)$, is a solution to the eigenvalue problem**

$$
\left[c_{i j k l}(x) n_{i} n_{l}-\rho(x) U^{2} \delta_{i k}\right] a_{k}=0 .
$$

Here the unit vector $n$ is the direction of propagation at $x$. Since the elasticity tensor obeys the symmetry relations

$$
c_{i j k l}=c_{k l i i},
$$

it is clear that (1.1) has three (not necessarily distinct) solutions $U_{1}, U_{2}, U_{3}$. More-

*Received March 13, 1964.

**We use indicial notation and Cartesian tensors throughout. Thus subscripts have the range $(1,2,3), x_{i}$ denote rectangular Cartesian coordinates, and $x=\left(x_{1}, x_{2}, x_{3}\right)$. Moreover summation over repeated subscripts is implied, $\delta_{i j}$ is Kronecker's delta, subscripts preceded by a comma indicate differentiation with respect to the corresponding Cartesian coordinate. 